\title{
Patients with normal-range CA19-9 levels represent a distinct subgroup of pancreatic cancer patients
}

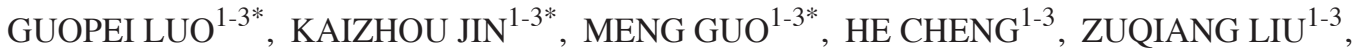 \\ ZHIWEN XIAO ${ }^{1-3}$, YU LU ${ }^{1-3}$, JIANG LONG ${ }^{1-3}$, LIANG LIU ${ }^{1-3}$, JIN XU ${ }^{1-3}$ CHEN LIU ${ }^{1-3}$, \\ YUTANG GAO ${ }^{4}$, QUANXING NI ${ }^{1-3}$ and XIANJUN YU ${ }^{1-3}$ \\ ${ }^{1}$ Department of Pancreatic Surgery, Fudan University Shanghai Cancer Center; ${ }^{2}$ Department of Oncology, \\ Shanghai Medical College of Fudan University; ${ }^{3}$ Pancreatic Cancer Institute, Fudan University; \\ ${ }^{4}$ Department of Epidemiology, Shanghai Cancer Institute, Shanghai 200032, P.R. China
}

Received August 6, 2016; Accepted October 26, 2016

DOI: 10.3892/ol.2016.5501

\begin{abstract}
Pancreatic cancer remains a lethal disease that responds poorly to multiple types of treatment. Therefore, the identification of distinct subgroups that exhibit unique therapeutic responses is an urgent requirement. In the present multicenter study (1,912 cases), the differences between the therapeutic responses and clinical characteristics of two subgroups of pancreatic cancer, carbohydrate antigen 19-9 (CA19-9)-normal (baseline serum level, $\leq 37 \mathrm{U} / \mathrm{ml}$ ) and CA19-9-elevated (baseline serum level, $>37 \mathrm{U} / \mathrm{ml}$ ), were analyzed. CA19-9-normal expression was identified to be an independent prognostic factor for patients with stage I-II [hazard ratio $(\mathrm{HR})=0.77 ; \mathrm{P}=0.037$ ] and stage III-IV $(\mathrm{HR}=0.68$; $\mathrm{P}<0.001)$ pancreatic cancer. The 5-year survival rate of the stage III-IV CA19-9-normal subgroup was increased compared with the stage I-II CA19-9-elevated subgroup (15.4 vs. 13.8\%). In the stage I-II CA19-9-normal and CA19-9-elevated subgroups, gemcitabine-based chemotherapy was a significant positive prognostic factor for survival (CA19-9-normal, $\mathrm{HR}=0.54, \mathrm{P}=0.013$; CA19-9-elevated, $\mathrm{HR}=0.55, \mathrm{P}<0.001)$. However, among stage III-IV patients, the CA19-9-normal subgroup exhibited a poor response to gemcitabine-based chemotherapy $(\mathrm{HR}=0.77 ; \mathrm{P}=0.165)$, while the CA19-9-elevated subgroup exhibited a favorable response, resulting in a lower rate of mortality $(\mathrm{HR}=0.70 ; \mathrm{P}<0.001)$ compared with no chemotherapy. It was concluded that CA19-9-normal pancreatic cancer is a less aggressive subgroup; however, advanced
\end{abstract}

Correspondence to: $\mathrm{Dr}$ Xianjun Yu, Department of Pancreatic Surgery, Fudan University Shanghai Cancer Center, 270 Dong'An Road, Shanghai 200032, P.R. China

E-mail: yuxianjun@fudanpci.org

\section{*Contributed equally}

Key words: pancreatic adenocarcinoma, subtype, Lewis, carbohydrate antigen 19-9, gemcitabine, chemotherapy
CA19-9-normal pancreatic cancer exhibits a poorer response to gemcitabine-based chemotherapy.

\section{Introduction}

Although marked progress in recent decades has been made in the treatment of cancer, pancreatic cancer remains a lethal disease, with a 5-year survival rate of $<6 \%(1,2)$. Personalized medicine and surgery is tailored to the individual patient, and has the potential to improve the management of pancreatic cancer $(3,4)$. As pancreatic cancer is a malignant tumor that exhibits heterogeneous biological characteristics, it may be susceptible to treatment with personalized medicine (5). Global genomic analyses have revealed various core signaling pathways in pancreatic cancer that may represent ideal targets for personalized treatment, including K-Ras, transforming growth factor $\beta$, c-Jun $\mathrm{N}$-terminal kinases, integrin, Wnt/Notch, Hedgehog, control of G1/S phase, apoptosis, DNA damage control, small GTPases, invasion and homophilic cell adhesion (4). It is necessary to identify distinct pancreatic cancer subgroups with unique characteristics in order to allow the selection of personalized treatments.

Carbohydrate antigen 19-9 (CA19-9) is a tumor-associated biomarker and its expression requires the presence of sialylated Lewis antigen (6-10). It has been extensively used as a pancreatic cancer biomarker at various phases of pancreatic cancer management $(6,8,9,11-13)$. The recommended upper limit for normal serum CA19-9 expression is $37 \mathrm{U} / \mathrm{ml}$, as determined by the standard deviation of CA19-9 expression in the normal population $(11,12,14)$. Several studies have demonstrated that early- and advanced-stage pancreatic cancer patients with normal serum CA19-9 expression $(\leq 37 \mathrm{U} / \mathrm{ml})$ had a significant survival advantage compared with patients with elevated serum CA19-9 expression (>37 U/ml) $(11,14,15)$. However, the clinical features of pancreatic cancer occurring with normal CA19-9 levels remain unknown.

In the present multicenter study, an extensive analysis of the clinical, pathological and biological features of patients with various stages of pancreatic cancer, who were stratified by normal and elevated baseline serum CA19-9 levels, was performed. 

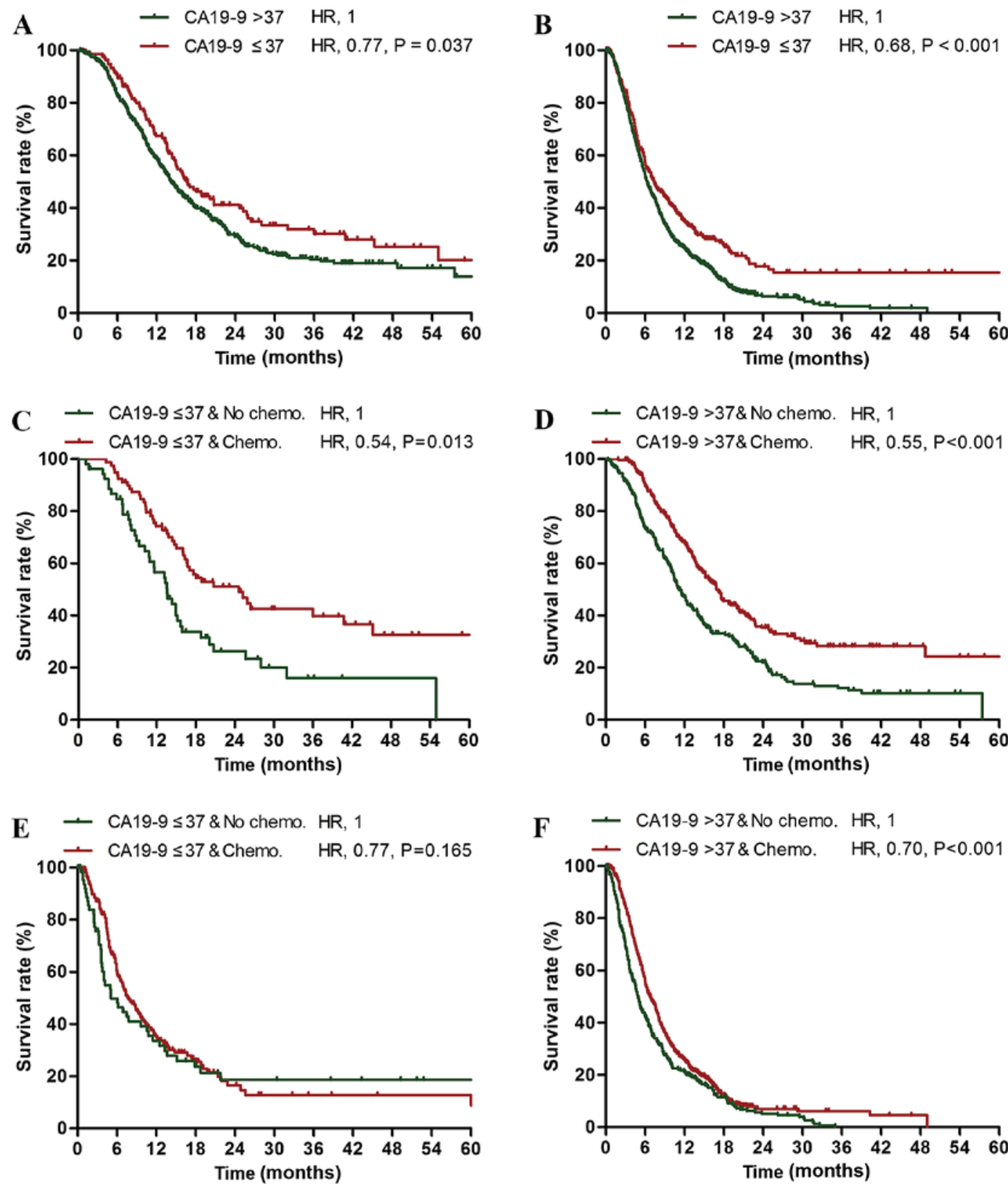

Figure 1.Kaplan-Meier estimators for patients with pancreatic cancer subdivided by baseline serum CA19-9 levels (CA19-9-normal, $\leq 37$ U/ml; CA19-9-elevated $>37 \mathrm{U} / \mathrm{ml}$ ), tumor stage and chemotherapy. Survival rates for patients with (A) stage I-II and (B) stage III-IV pancreatic cancer were significantly improved in the CA19-9-normal subgroups compared with the CA19-9-elevated subgroups. Among stage I-II patients, survival rates were significantly improved by treatment with gemcitabine in (C) CA19-9-normal and (D) CA19-9-elevated subgroups. Among stage III-IV patients, survival rates were not significantly improved by gemcitabine treatment in (E) the CA19-9-normal subgroup, but were in (F) the CA19-9-elevated subgroup. CA19-9, carbohydrate antigen 19-9; HR, hazard ratio; chemo., chemotherapy.

\section{Materials and methods}

Patients. All patients (1,912 cases) were selected from a multicenter database constructed by the Shanghai Cancer Center of Fudan University and the Shanghai Cancer Institute (Shanghai, China); patients treated between December 2006 and March 2016 were included. The protocol used in the present study conformed to the ethical guidelines of The Declaration of Helsinki and was approved by the Ethics Boards of the Shanghai Cancer Institute and Shanghai Cancer Center. Written informed consent was obtained from all patients participating in the study. Patients were stratified according to their baseline serum CA19-9 level and type of treatment received (surgery, chemotherapy, radiotherapy or best supportive care). Survival time was calculated as the time between the date of diagnosis and the date of the latest follow-up or mortality (14). Follow-up information was updated in April 2016.

The included patients were those who had histological or cytological evidence of pancreatic adenocarcinoma. Exclusion criteria included endocrine or acinar pancreatic carcinoma, or intraductal papillary mucinous neoplasm associated pancreatic adenocarcinoma. Patients lacking detailed information for serum CA19-9 levels were also excluded. Tumors were staged according to the 7th edition of the American Joint Committee on Cancer (Chicago, IL, USA) classification (16). All patients 
Table I. Multivariate analysis (Cox proportional hazards model) of the association between mortality and the clinical characteristics of patients with stage I-II and stage III-IV pancreatic cancer.

\begin{tabular}{|c|c|c|c|c|}
\hline \multirow[b]{2}{*}{ Clinical characteristics } & \multicolumn{2}{|c|}{ Stage I-II ${ }^{\mathrm{a}}$} & \multicolumn{2}{|c|}{ Stage III-IV ${ }^{b}$} \\
\hline & HR & P-value & HR & P-value \\
\hline Age ( $>60$ vs. $\leq 60$ years $)$ & 1.14 & 0.217 & 1.15 & 0.037 \\
\hline Gender (female vs. male) & 0.94 & 0.548 & 0.93 & 0.270 \\
\hline Tumor location (pancreatic head vs. all others) & 1.13 & 0.255 & 1.09 & 0.194 \\
\hline ECOG performance status (2-3 vs. $0-1)$ & l & 1 & 1.65 & $<0.001$ \\
\hline Tumor size ( $>4$ vs. $\leq 4 \mathrm{~cm})$ & 1.79 & $<0.001$ & 1.15 & 0.042 \\
\hline Nerve invasion status (positive vs. negative) & 1.89 & 0.001 & / & l \\
\hline Vessel invasion status (positive vs. negative) & 1.59 & 0.002 & / & / \\
\hline Lymph node metastasis status (positive vs. negative) & 1.67 & $<0.001$ & l & / \\
\hline Tumor grade (high vs. low) & 1.32 & 0.012 & l & l \\
\hline Distant metastasis status (positive vs. negative) & / & / & 1.93 & $<0.001$ \\
\hline Chemotherapy (administered vs. not administered) & 0.58 & 0.001 & 0.71 & $<0.001$ \\
\hline CA19-9 level ( $\leq 37$ vs. $>37 \mathrm{U} / \mathrm{ml})$ & 0.77 & 0.037 & 0.68 & $<0.001$ \\
\hline
\end{tabular}

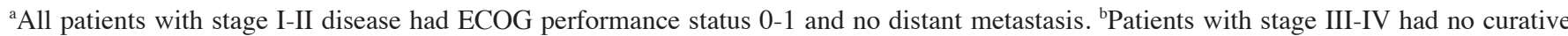
resection and no information regarding nerve and vessel invasion, lymph node metastasis or tumor grade. HR, hazard ratio; ECOG, Eastern Cooperative Oncology Group; CA19-9, carbohydrate antigen 19-9; /, not evaluated.

with stage I or II pancreatic cancer received curative-intent resection. Although serum CA19-9 levels have been documented to be affected by altered biliary excretion, such as with biliary tract obstruction (9), this effect was ignored as the subjects in this study were subdivided into subgroups with CA19-9 levels $\leq 37 \mathrm{U} / \mathrm{ml}$ (CA19-9-normal) and $\geq 37 \mathrm{U} / \mathrm{ml}$ (CA19-9-elevated).

Statistical analysis. Continuous variables are presented as the mean \pm standard deviation. Time-to-event variables and the 2- and 5-year survival rates were examined using the Kaplan-Meier estimator. The treatment arms were compared using log-rank tests, and stratified by serum CA19-9 levels and type of treatment received. Multivariate analysis of the association between mortality and the clinical characteristics of patients was performed by Cox proportional hazards model. Continuous variables were compared using the Student's t-test or the Wilcoxon rank-sum test, where appropriate. Dichotomous variables were compared using the $\chi^{2}$ test. STATA statistical software package (version 12.0; StataCorp LP, College Station, TX, USA) was used for all analyses. $\mathrm{P}<0.05$ was considered to indicate a statistically significant difference.

\section{Results}

Baseline characteristics. The present study analyzed a total of 1,912 cases (588 of stage I-II and 1,324 of stage III-IV), of which the median survival time was 8.7 months and the 5 -year survival rate was $8.0 \%$. A total of $80.4 \%$ of patients exhibited baseline serum CA19-9 levels of $>37 \mathrm{U} / \mathrm{ml}$. No statistically significant differences in patient age $(\mathrm{P}=0.495)$, gender $(\mathrm{P}=0.670)$, tumor location $(\mathrm{P}=0.215)$, tumor size $(\mathrm{P}=0.352)$, tumor grade $(\mathrm{P}=0.947)$, lymph node metastasis
$(\mathrm{P}=0.166)$, nerve invasion status $(\mathrm{P}=0.656)$ or vessel invasion status $(\mathrm{P}=0.863)$ were observed between the CA19-9-normal and CA19-9-elevated subgroups in patients of stage I-II. In addition, no statistically significant differences in patient age $(\mathrm{P}=0.077)$, gender $(\mathrm{P}=0.489)$, Eastern Cooperative Oncology Group (ECOG) performance status $(\mathrm{P}=0.762)$, tumor location $(\mathrm{P}=0.487)$, tumor size $(\mathrm{P}=0.119)$ or distant metastasis status $(\mathrm{P}=0.939)$ were observed between the CA19-9-normal and CA19-9-elevated subgroups in patients of stage III-IV.

Improved prognosis in the CA19-9-normal subgroup. Among the patients with stage I-II cancer, the CA19-9-normal subgroup exhibited an increased survival rate compared with the CA19-9-elevated subgroup (median survival times, 16.6 vs. 14.2 months; 2-year survival rates, 39.9 vs. $29.1 \%$; Fig. 1A). In addition, among the stage III-IV patients, the CA19-9-normal subgroup exhibited an increased survival rate compared with the CA19-9-elevated subgroup (median survival times, 7.4 vs. 6.3 months; 2 -year survival rates, 17.6 vs. $6.3 \%$; Fig. 1B). The 5-year survival rate of the stage III-IV CA19-9-normal subgroup was increased compared with the stage I-II CA19-9-elevated subgroup (15.4 vs. 13.8\%; Fig. 1A and B). Normal serum CA19-9 level was identified to be an independent prognostic factor for mortality in patients with stage I-II and stage III-IV pancreatic cancer [stage I-II, hazard ratio $(\mathrm{HR})=0.77, \mathrm{P}=0.037$; stage $\mathrm{III}-\mathrm{IV}, \mathrm{HR}=0.68, \mathrm{P}<0.001$; Table I].

Efficacy of gemcitabine-based chemotherapy. Patients with stage I-II pancreatic cancer who underwent neoadjuvant therapy, adjuvant radiotherapy or non-gemcitabine-based adjuvant chemotherapy were excluded from the evaluation of the response to gemcitabine-based chemotherapy. 
Table II. Association between mortality and the clinical characteristics of patients with stage I-II pancreatic cancer stratified by baseline CA19-9 levels according to Cox proportional hazards model.

\begin{tabular}{lcccrr}
\hline & \multicolumn{2}{c}{ CA19-9 $\leq 37 \mathrm{U} / \mathrm{ml}$} & & \multicolumn{2}{c}{ CA19-9 $>37 \mathrm{U} / \mathrm{ml}$} \\
\cline { 2 - 3 } \cline { 5 - 6 } Clinical characteristics & HR & P-value & & HR & P-value \\
\hline Age $(>60$ vs. $\leq 60$ years) & 1.37 & 0.198 & & 1.08 & 0.528 \\
Gender (female vs. male) & 0.66 & 0.103 & 1.00 & 0.983 \\
Tumor location (pancreatic head vs. all others) & 1.25 & 0.396 & 1.10 & 0.439 \\
Tumor size ( $>4$ vs. $\leq 4$ cm) & 2.13 & 0.046 & 1.75 & 0.001 \\
Nerve invasion status (positive vs. negative) & 2.50 & 0.052 & 1.83 & 0.004 \\
Vessel invasion status (positive vs. negative) & 2.55 & 0.019 & & 1.56 & 0.007 \\
Lymph metastasis status (positive vs. negative) & 0.66 & 0.234 & & 1.97 & $<0.001$ \\
Tumor grade (high vs. low) & 1.39 & 0.202 & 1.33 & 0.024 \\
Chemotherapy (administered vs. not administered) & 0.54 & 0.013 & 0.55 & $<0.001$ \\
\hline
\end{tabular}

HR, hazard ratio; CA19-9, carbohydrate antigen 19-9.

Table III. Association between the mortality rate and clinical characteristics of patients with stage III-IV pancreatic cancer according to Cox proportional hazards model.

\begin{tabular}{llccrr}
\hline & \multicolumn{2}{c}{ CA19-9 $\leq 37 \mathrm{U} / \mathrm{ml}$} & & \multicolumn{2}{c}{ CA19-9 $>37 \mathrm{U} / \mathrm{ml}$} \\
\cline { 5 - 6 } Clinical characteristics & HR & P-value & & HR & P-value \\
\hline Age $(>60$ vs. $\leq 60$ years) & 1.44 & 0.028 & & 1.10 & 0.173 \\
Gender (female vs. male) & 1.01 & 0.954 & & 0.92 & 0.245 \\
Tumor location (pancreatic head vs. all others) & 0.93 & 0.687 & & 1.13 & 0.093 \\
ECOG performance status (0-1 vs. 2-3) & 0.54 & 0.005 & & 0.68 & $<0.001$ \\
Tumor size ( $>4$ vs. $\leq 4$ cm) & 1.17 & 0.377 & & 1.18 & 0.035 \\
Distant metastasis status (positive vs. negative) & 1.88 & 0.001 & & 1.96 & $<0.001$ \\
Chemotherapy (administered vs. not administered) & 0.77 & 0.165 & & 0.70 & $<0.001$ \\
\hline
\end{tabular}

HR, hazard ratio; ECOG, Eastern Cooperative Oncology Group; CA19-9, carbohydrate antigen 19-9.

In addition, patients with stage III-IV pancreatic cancer who underwent radiotherapy or non-gemcitabine based chemotherapy were excluded from the evaluation of response to gemcitabine-based chemotherapy. No statistically significant differences were observed in the proportion of gemcitabine-based chemotherapy administered in the stage I-II CA19-9-normal subgroup compared with the stage I-II CA19-9-elevated subgroup (59.8 vs. 55.7\%; $\mathrm{P}=0.397$ ), or in the stage III-IV CA19-9-normal subgroup compared with the stage III-IV CA19-9-elevated subgroup (71.9 vs. $72.0 \%$; $\mathrm{P}=0.976$ ). The stage I-II CA19-9-normal $(\mathrm{HR}=0.54 ; \mathrm{P}=0.013)$ and $\mathrm{CA} 19-9$-elevated $(\mathrm{HR}=0.55$; $\mathrm{P}<0.001)$ subgroups exhibited significantly increased survival rates following treatment with gemcitabine compared with the untreated subgroups (Table II; Fig. 1C and D). However, the stage III-IV CA19-9-normal subgroup exhibited no significant change in survival rate following treatment with gemcitabine compared with the untreated subgroup (HR=0.77; $\mathrm{P}=0.165$; Table III; Fig. 1E), while the stage III-IV CA19-9-elevated subgroup exhibited a significant increase in the 2-year and
5 -year survival rates following treatment with gemcitabine compared with the untreated subgroup $(\mathrm{HR}=0.70 ; \mathrm{P}<0.001$; Table III; Fig. 1F) sp16.

\section{Discussion}

CA19-9 is the most important tumor marker in pancreatic cancer and is aberrantly secreted by the majority of pancreatic tumors $(6,9,11-15,17,18)$. However, a distinct subset of patients with pancreatic cancer present with normal serum CA19-9 levels and are occasionally Lewis antigen-positive. These patients exhibit decreased or no CA19-9 secretion, independent of Lewis antigen genotype (18). In the present study, patients with CA19-9-normal pancreatic cancer were demonstrated to be a distinct subgroup that has a more favorable prognosis and unique therapeutic response.

The improved prognosis and distinct therapeutic response of the CA19-9-normal subgroup cannot be attributed to tumor burden or stage, but to its biological behavior. CA19-9, also known as sialylated Lewis a antigen, has been reported to 
promote metastasis by binding E-selectin, which is expressed on the surface of endothelial cells $(7,19)$. Several studies have demonstrated that CA19-9 promotes pancreatic cancer cell metastasis (20-22), suggesting that CA19-9 is a therapeutic target in the treatment of pancreatic cancer. However, further studies are required to confirm this hypothesis.

Chemotherapy is frequently utilized in the treatment of pancreatic cancer. In the present study, it was observed that patients with stage I-II pancreatic cancer exhibited a significantly increased survival rate following gemcitabine-based adjuvant chemotherapy, compared with the untreated patients. In addition, gemcitabine-based chemotherapy was effective against stage III-IV pancreatic tumors with elevated CA19-9 expression. However, patients with stage III-IV CA19-9-normal pancreatic cancer exhibited a poor response to gemcitabine-based chemotherapy. Novel chemotherapeutic agents and regimens are required for the treatment of advanced stage pancreatic cancer with normal CA19-9 expression.

The present study did not determine the clinical and pathological characteristics of Lewis antigen-negative patients with pancreatic cancer. However, several studies have observed similar survival rates in patients with resectable pancreatic adenocarcinoma with undetectable and normal CA19-9 levels $(14,15,23)$. Furthermore, abnormal Lewis antigens (including types $a$ and $b$ ) and the Lewis enzyme have been detected in normal and neoplastic tissue samples from patients typed as Lewis antigen $\mathrm{a}^{-} \mathrm{b}^{-}$on red blood cells (18,24-27). For example, Orntoft et al (27) detected Lewis antigens in $3 / 6$ cancer-bearing patients using immunohistology and immunochemistry; however all 6 patients were typed as Lewis antigen $\mathrm{a}^{-} \mathrm{b}^{-}$according to hemagglutination assays. These observations further support that normal serum CA19-9 expression should be viewed as a distinct subgroup of pancreatic cancer, independently of Lewis antigen status.

In conclusion, CA19-9-normal pancreatic cancer is a less aggressive subgroup of pancreatic cancer that has distinct clinical, pathological and biological characteristics. The characterization of this subgroup may have a great impact on the overall management of pancreatic cancer. Clinical trials should be separately conducted on patients with pancreatic cancer who are subdivided by baseline serum CA19-9 levels. Despite its large sample size, the present study is limited by its retrospective nature; therefore prospective randomized clinical studies are required to confirm the results.

\section{Acknowledgements}

The authors would like to thank Professor Jianfeng Luo from the School of Public Health of Fudan University (Shanghai, China), Dr Zengying Liu and Dr Jing Huang from Life Technologies Comapny (Carlsbad, CA, USA), Dr Jing Wang from the Shanghai Cancer Institute, Professor Dong Xiang from the Shanghai Blood Center (Shanghai, China), and Professor Menghong Sun from the Tissue Bank of the Shanghai Cancer Center, for their technical assistance. The present study was supported by the Shanghai Rising Star Program (grant no. 14QA1400900) and the National Science Foundation of China (grant no. 81372649).

\section{References}

1. Hidalgo M: Pancreatic cancer. N Engl J Med 362: 1605-1617, 2010.

2. Siegel R, Naishadham D and Jemal A: Cancer statistics, 2012. CA Cancer J Clin 62: 10-29, 2012.

3. Hamburg MA and Collins FS: The path to personalized medicine. N Engl J Med 363: 301-304, 2010.

4. Ko AH and Tempero MA: Personalized medicine for pancreatic cancer: A step in the right direction. Gastroenterology 136: 43-45, 2009.

5. Jones S, Zhang X, Parsons DW, Lin JC, Leary RJ, Angenendt P, Mankoo P, Carter H, Kamiyama H, Jimeno A, et al: Core signaling pathways in human pancreatic cancers revealed by global genomic analyses. Science 321: 1801-1806, 2008.

6. Goonetilleke KS and Siriwardena AK: Systematic review of carbohydrate antigen (CA 19-9) as a biochemical marker in the diagnosis of pancreatic cancer. Eur J Surg Oncol 33: 266-270, 2007.

7. Kannagi R: Carbohydrate antigen sialyl Lewis a-its pathophysiological significance and induction mechanism in cancer progression. Chang Gung Med J 30: 189-209, 2007.

8. Pleskow DK, Berger HJ, Gyves J, Allen E, McLean A and Podolsky DK: Evaluation of a serologic marker, CA19-9, in the diagnosis of pancreatic cancer. Ann Intern Med 110: 704-709, 1989.

9. Schlieman MG, Ho HS and Bold RJ: Utility of tumor markers in determining resectability of pancreatic cancer. Arch Surg 138: 951-955, 2003.

10. Tempero MA, Uchida E, Takasaki H, Burnett DA, Steplewski Z and Pour PM: Relationship of carbohydrate antigen 19-9 and Lewis antigens in pancreatic cancer. Cancer Res 47: 5501-5503, 1987.

11. Ferrone CR, Finkelstein DM, Thayer SP, Muzikansky A, Fernandez-delCastillo C and Warshaw AL: Perioperative CA19-9 levels can predict stage and survival in patients with resectable pancreatic adenocarcinoma. J Clin Oncol 24: 2897-2902, 2006.

12. Humphris JL, Chang DK, Johns AL, Scarlett CJ, Pajic M, Jones MD, Colvin EK, Nagrial A, Chin VT, Chantrill LA, et al: The prognostic and predictive value of serum CA19.9 in pancreatic cancer. Ann Oncol 23: 1713-1722, 2012.

13. Kondo N, Murakami Y, Uemura K, Hayashidani Y, Sudo T, Hashimoto Y, Nakashima A, Sakabe R, Shigemoto N, Kato Y, et al: Prognostic impact of perioperative serum CA 19-9 levels in patients with resectable pancreatic cancer. Ann Surg Oncol 17: 2321-2329, 2010.

14. Berger AC, Garcia M Jr, Hoffman JP, Regine WF, Abrams RA, Safran H, Konski A, Benson AB III, MacDonald J and Willett CG: Postresection CA 19-9 predicts overall survival in patients with pancreatic cancer treated with adjuvant chemoradiation: A prospective validation by RTOG 9704 . J Clin Oncol 26: 5918-5922, 2008.

15. Berger AC, Meszoely IM, Ross EA, Watson JC and Hoffman JP: Undetectable preoperative levels of serum CA 19-9 correlate with improved survival for patients with resectable pancreatic adenocarcinoma. Ann Surg Oncol 11: 644-649, 2004.

16. Edge SB and Compton CC: The American Joint Committee on Cancer: The 7th edition of the AJCC cancer staging manual and the future of TNM. Ann Surg Oncol 17: 1471-1474, 2010.

17. Magnani JL, Steplewski Z, Koprowski H and Ginsburg V: Identification of the gastrointestinal and pancreatic cancer-associated antigen detected by monoclonal antibody 19-9 in the sera of patients as a mucin. Cancer Res 43: 5489-5492, 1983.

18. Luo G, Guo M, Jin K, Liu Z, Liu C, Cheng H, Lu Y, Long J, Liu L, $\mathrm{Xu}$ J, et al: Optimize CA19-9 in detecting pancreatic cancer by Lewis and Secretor genotyping. Pancreatology pii: S1424-3903, 2016 (Epub ahead of print).

19. Takada A, Ohmori K, Yoneda T, Tsuyuoka K, Hasegawa A, Kiso $\mathrm{M}$ and Kannagi R: Contribution of carbohydrate antigens sialyl Lewis A and sialyl Lewis X to adhesion of human cancer cells to vascular endothelium. Cancer Res 53: 354-361, 1993.

20. Aubert M, Panicot L, Crotte C, Gibier P, Lombardo D, Sadoulet MO and Mas E: Restoration of alpha $(1,2)$ fucosyltransferase activity decreases adhesive and metastatic properties of human pancreatic cancer cells. Cancer Res 60: 1449-1456, 2000.

21. Aubert M, Panicot-Dubois L, Crotte C, Sbarra V, Lombardo D, Sadoulet MO and Mas E: Peritoneal colonization by human pancreatic cancer cells is inhibited by antisense FUT3 sequence. Int J Cancer 88: 558-565, 2000. 
22. Iwai K, Ishikura H, Kaji M, Sugiura H, Ishizu A, Takahashi C, Kato H, Tanabe T and Yoshiki T: Importance of E-selectin (ELAM-1) and sialyl Lewis(a) in the adhesion of pancreatic carcinoma cells to activated endothelium. Int J Cancer 54: 972-977, 1993.

23. Hartwig W, Strobel O, Hinz U, Fritz S, Hackert T, Roth C, Büchler MW and Werner J: CA19-9 in potentially resectable pancreatic cancer: Perspective to Adjust Surgical and Perioperative Therapy. Ann Surg Oncol 20: 2188-2196, 2013.

24. Björk S, Breimer ME, Hansson GC, Karlsson KA and Leffler $\mathrm{H}$ Structures of blood group glycosphingolipids of human small intestine. A relation between the expression of fucolipids of epithelial cells and the ABO, Le and Se phenotype of the donor. J Biol Chem 262: 6758-6765, 1987.
25. Hamada E, Taniguchi T, Baba S and Maekawa M: Investigation of unexpected serum CA19-9 elevation in Lewis-negative cancer patients. Ann Clin Biochem 49: 266-272, 2012.

26. Hirano K, Kawa S, Oguchi H, Kobayashi T, Yonekura H, Ogata $\mathrm{H}$ and Homma T: Loss of Lewis antigen expression on erythrocytes in some cancer patients with high serum CA19-9 levels. J Natl Cancer Inst 79: 1261-1268, 1987.

27. Orntoft TF, Holmes EH, Johnson P, Hakomori S and Clausen H: Differential tissue expression of the Lewis blood group antigens: Enzymatic, immunohistologic, and immunochemical evidence for Lewis $a$ and $b$ antigen expression in Le(a-b-) individuals. Blood 77: 1389-1396, 1991. 